\title{
A Particle Swarm Optimization Algorithm for Scheduling Against Restrictive Common Due Dates
}

\author{
Andreas C. Nearchou* \\ Department of Business Administration, University of Patras, \\ 26500 Patras, Greece \\ Sotiris L. Omirou \\ Department of Mechanical Engineering, Frederick University, \\ Pallouriotissa 1036, Nicosia, Cyprus, \\ E-mail: $\underline{\text { eng.os@frederick.ac.cy }}$ \\ Received 1 April 2011 \\ Accepted 14 December 2012
}

\begin{abstract}
:
Focusing on the just-in-time (JIT) operations management, earliness as well as, tardiness of jobs' production and delivery should be discouraged. In accordance to this philosophy, scheduling problems involving earliness and tardiness penalties are very critical for the operations manager. In this paper, a new population heuristic based on the particle swarm optimization (PSO) technique is presented to solve the single machine early/tardy scheduling problem against a restrictive common due date. This type of scheduling sets costs depending on whether a job finished before (earliness), or after (tardiness) the specified due date. The objective is to minimize a summation of earliness and tardiness penalty costs, thus pushing the completion time of each job as close as possible to the due date. The problem is known to be $N P$-hard, and therefore large size instances cannot be addressed by traditional mathematical programming techniques. The performance of the proposed PSO heuristic is measured over benchmarks problems with up to 1000 jobs taken from the open literature, and found quite high and promising in respect to the quality of the solutions obtained. Particularly, PSO was found able to improve the $82 \%$ of the existing best known solutions of the examined benchmarks test problems.
\end{abstract}

Key words: Meta-heuristics, swarm intelligence, combinatorial optimization, job scheduling, just-in-time.

"Corresponding author. E-mail address: nearchou@upatras.gr 


\section{Introduction}

Sequencing and scheduling problems involving due dates, play a crucial role in real-world production and operations management. This type of scheduling sets penalty costs depending on whether a job finished before (earliness), or after (tardiness) the specified due date. In the last two decades, much research effort has been spent on the study of earliness and tardiness penalties in scheduling ${ }^{1,2}$ due to its accordance with the principles of just-in-time (JIT) operations management. JIT adopts the notion that jobs must be completed as close as possible to their due date, neither too early, nor too late. Early jobs result in inventory holding costs, while late jobs result to penalties such as loss of customer goodwill and loss of orders. Therefore, earliness as well as tardiness of jobs should be discouraged.

This paper deals with the single-machine early/tardy scheduling problem (SMETSP) of a set of jobs with a common due date (CDD) and objective the minimization of the jobs' total earliness and tardiness. SMETSP belongs to a large class of scheduling problems $^{2}$ formally classified as $\mathrm{n} / 1 / / \mathrm{ET}$. This class consists of problems with distinct due dates, problems with a CDD, problems with single, or multiple performance measures being either linear or non-linear, etc. Some of these problems can be solved in polynomial time using traditional mathematical programming methods, while many other are known to be intractable. One assumption often made about CDD is that it is sufficiently large so that it does not constrain the scheduling of the jobs. This is known as the unrestricted version of the problem (consequently CDD is called unrestrictive). The restricted version of the problem is obviously harder since the value of CDD is small enough to constrain the scheduling process.

Even the simplest formulation of SMETSP leads to an $N P$-hard combinatorial optimization problem $(\mathrm{COP})^{1,2}$, and thus it seems fair, large size instances of the problem to be addressed by the means of heuristics. Since the pioneer work of $\mathrm{Kanet}^{3}$ which deals exclusively with the special case when the earliness and tardiness penalties are equal to one, many approximate algorithms have been proposed for various versions of the basic problem. Hall ${ }^{4}$ and Bagchi et al. ${ }^{5,6}$ proposed algorithms for the absolute deviation SMETSP, which involve minimizing the sum of absolute deviations of the job completion times from a CDD. Hall and Posner ${ }^{7}$ examined the unrestricted weighted earliness and tardiness problem. De et $a l^{8}{ }^{\text {, }}$, proposed a greedy randomized adaptive heuristic for the unrestricted problem with different penalties to find both the optimal due date and the optimal sequence of the jobs. Hoogeveen and van de Velde ${ }^{9}$ addressed the unrestricted SMETSP with 'almost' CDD using a dynamic programming algorithm. More about algorithms on scheduling problems involving due dates can be found in two comprehensive reviews considered by Baker and Scudder ${ }^{1}$ and Cheng and Gupta ${ }^{10}$ respectively. These reviews cover the results published before 1990. Recent material and results can be found in the survey paper of Gordon et al. ${ }^{11}$.

The majority of the proposed algorithms for SMETSP addressed instances of the problem with a small number of jobs, up to 25 or 50 jobs. For instance, Abdul-Razaq and Potts ${ }^{12}$ solved to optimality problems with up to 25 jobs using a branch and bound algorithm. Souza and Wolsey ${ }^{13}$ proposed branch and bound algorithms for solving a class of four different scheduling problems (including SMETSP) with 20 and 30 jobs. Almeida and Centeno ${ }^{14}$ addressed SMETSP with up to 50 jobs via a composite algorithm that combines steepest-descent, simulated annealing and tabu-search. Recently, the use of meta-heuristics such as tabu-search (see Refs. 1517), genetic algorithms ${ }^{15}$, simulated annealing ${ }^{18}$, differential evolution ${ }^{19,20}$, ant colony optimization ${ }^{21-23}$, artificial immune systems ${ }^{24}$ enable researchers to address effectively large size instances of the problem. Among them, James ${ }^{17}$, Feldmann and Biskup ${ }^{18}$, Nearchou and Omirou ${ }^{19}$, Nearchou ${ }^{20}$, and Lee et al. ${ }^{22}$ addressed the restricted SMETSP with general earliness and tardiness penalties. Moreover, Biskup and Feldman ${ }^{25}$ generated a set of benchmarks for SMETSP together with their upper bounds on the optimal objective functions.

The current paper investigates the application of the particle swarm optimizer (PSO) algorithm on the 
restricted SMETSP with general earliness and tardiness penalties. PSO is one of the latest meta-heuristics introduced by Eberhart and Kennedy ${ }^{26}$ for optimization over continuous spaces and its application to discrete COPs is still limited. Previous PSO-based approaches on single-machine scheduling problems can be found in the recent works of Anghinolfi and Paolucci ${ }^{27}$ and Low et $a .^{28}$. The former work tackled the classical singlemachine weighed tardiness scheduling problem with sequence-dependent setup times; while, the latter considered the single-machine scheduling problem with periodic maintenance. To the best of our knowledge, the only researchers who addressed scheduling problems involving due dates using the PSO algorithm have been Pan et $a .^{29}$. Our PSO approach differs from these studies in two main points: first in the developed encoding mechanism; that is, in the way 'particles' are represented and mapped into actual scheduling solutions; and second, in the way of controlling the 'particles' velocities of the entire swarm.

The performance of the proposed PSO algorithm is examined over the most restricted instances against CDDs of the Biskup and Feldmann (2001)'s benchmarks ${ }^{25}$; including 140 instances in total ranging from 10 to 1000 jobs. The results obtained are of high quality since new upper bounds have been introduced by PSO in the $82 \%$ of the examined benchmarks instances. The motivation behind the idea of applying PSO on SMETSP goes back to our previous works ${ }^{19,20}$ in which we tackled the problem with high success through another meta-heuristic namely, differential evolution. Since the last five years there is a considerably research interest to tackle hard COPs through PSO-based approaches, it was decided to investigate the behavior and the performance of PSO on SMETSP too. Therefore, a major objective of this work is to show (through extended analysis of critical aspects, such as solution space, representation, encoding and decoding mechanisms) how PSO, a metaheuristic initially proposed as global optimizer over continuous search spaces can be applied with success on discrete COPs too.

The rest of this paper is organized as follows: Section 2 states the problem. Section 3 gives a description of the basic PSO algorithm for optimization over continuous search spaces. Section 4 introduces the way PSO can be applied on SMETSP (a typical discrete
$\mathrm{COP}$ ), while Section 5 presents and discusses the results of the experimental evaluations of the algorithm. Finally, Section 6 summarizes the contribution of the paper and states some directions for future work.

\section{Problem formulation}

SMETSP can be formally defined as follows: consider $n$ jobs (numbered $1,2, \ldots, n$ ) to be processed without interruption on a single machine that can handle only one job at a time. Each job $j(j=1, \ldots, n)$ is available at time zero, requires a positive processing time $p_{j}$ and ideally must be completed exactly on a specific (common for all jobs) due date $d$. Penalties are incurred whenever a job is completed before or after this due date. Therefore, an ideal schedule is one in which all jobs finish on the specific due date. Assuming that $C_{j}$ is the completion time of job $j$, then the earliness and tardiness of job $j$ are given by the relations, $E_{j}=\max \left(0, d-C_{j}\right) \quad$ and $T_{j}=\max \left(0, C_{j}-d\right)$, respectively, for all $j=1, \ldots, n$. The objective is therefore to find a processing order of the $n$ jobs that minimizes

$$
\sum_{j=1}^{n}\left(\alpha_{j} E_{j}+\beta_{j} T_{j}\right)
$$

where $\alpha_{j}, \beta_{j}(j=1, \ldots, n)$ are the earliness and tardiness (nonnegative) penalties, respectively, for job $j$ and constitute data input to the scheduling problem.

Penalties in Eq. (1) can be measured in different ways resulting in several variations of the basic SMETSP. A CDD $d$ is called unrestrictive when $d \geq \sum p_{j}(j=1, \ldots, n)$ holds, otherwise is called restrictive. Moreover, $d$ is also called unrestrictive when it constitutes a decision variable for the problem. Consequently, one can refer to the problem as either unrestricted or restricted SMETSP.

The basic assumptions with SMETSP can be summarized as follows:

B Jobs' processing times are deterministic.

B Machine breakdown and maintenance are neglected. The machine is continuously available and never kept idle while there are jobs waiting to proceed.

B The machine processes only one job at a time.

B No setups between jobs are assumed.

B Jobs are known in advance. 
B Each job is available for processing at time zero.

B No job pre-empt is permitted.

B Jobs are independent without precedence or other constraints.

B All jobs must be completed on a particular common due date.

\subsection{Properties for the unrestricted CDD SMETSP}

There exists an optimal solution to the unrestricted CDD SMETSP having the following properties:

a) There is no inserted idle time in the schedule.

b) The schedule, is V-shaped (see Fig. 1), i.e., early jobs are sequenced in non-increasing order of $p_{j} / \alpha_{j}$ ('\-shaped' format), and late jobs are sequenced in non-decreasing order of $p_{j} / \beta_{j}$ $(j=1, \ldots, n)($ '/-shaped' format).

c) One job is completed exactly on the due date.

d) The $q$-th job in the sequence completes on the duedate $d$, where $q$ is the smallest integer satisfying the inequality $\sum_{j=1}^{q}\left(\alpha_{j}+\beta_{j}\right) \geq \sum_{j=1}^{n} \beta_{j}$

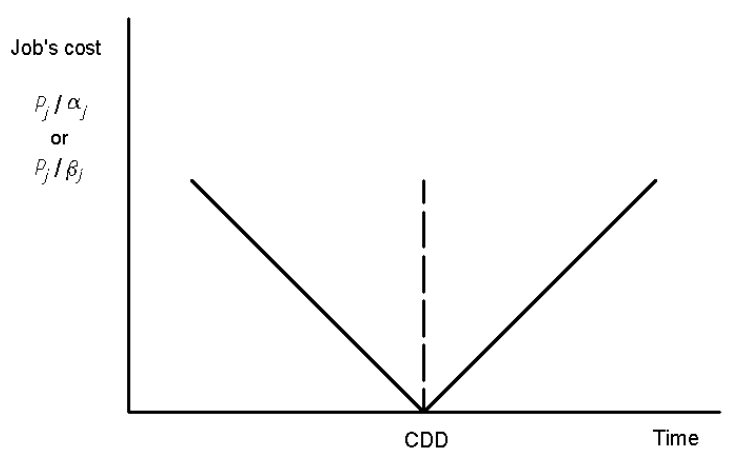

Fig. 1: The V-shaped property

\subsection{Properties for the restricted CDD SMETSP}

For the restricted SMETSP with general earliness and tardiness penalties there is an optimal schedule with the following properties:

a) No idle times are inserted between consecutive jobs $^{30}$.

b) The schedule is V-shaped, but a straddling job may exist, i.e., a job whose execution starts before and finished after the due date ${ }^{25}$. c) The processing time of the first job either starts at time zero, or one job is completed at the due date ${ }^{25}$.

Obviously, the restricted SMETSP is much more complex than the unrestricted version since all its variations result to an $N P$-hard $\mathrm{COP}^{11}$. There is a gap in the related literature for algorithms taking into account the third property of the restricted SMETSP. That is, the case where the first job in an optimal schedule might not start at time zero; thus, excluding optimal schedules a priori. This characteristic is investigated in Biskup and Feldmann ${ }^{25}$. It is worth pointing out that, there is no such limitation with the proposed PSO algorithm. Particularly, the use of a suitable encoding scheme enables PSO taking into account the case of the third property in the generated SMETSP solutions.

\section{The particle swarm optimization (PSO) algorithm}

PSO is a stochastic population heuristic introduced by Eberhart and Kennedy ${ }^{26}$ for continuous non-linear function optimization. According to its founders, PSO has roots in two recent 'intelligent' optimization methodologies: in artificial life and in evolutionary computation. In regard to artificial life, PSO has ties with bird flocking and fish schooling theories, while in regard to evolutionary computation has similarities with genetic and evolutionary algorithms. Since its invention, PSO has been applied with success on various COPs such as, the unit commitment problem ${ }^{31}$, the traveling salesman problem ${ }^{32}$, the task assignment problem $^{33}$, an optimal operational path finding for automated drilling operations ${ }^{34}$, a multi-objective order planning production problem in steel sheets manufacturing $^{35}$, scheduling problems involving duedates $^{29}$, the shortest path problem ${ }^{36}$, etc. Recently, its application has been extended on scheduling problems such as, flow-shop scheduling problems ${ }^{37-41}$, the singlemachine total weighting tardiness problem ${ }^{27,42}$, the single machine scheduling problem with periodic maintenance ${ }^{28}$, the two-stage assembly-scheduling problem $^{43}$, and job-shop scheduling problems ${ }^{44,45}$.

Assuming the problem of minimizing a real-valued function $f(x), x \in \Omega \subset \Re^{D}$ ( $\Omega$ is assumed to be the feasible search space of the problem), PSO utilizes a set (called swarm) of $N s$ particles as a population to search 
$\Omega$ toward the global optimal solution. Each particle represents a potential solution to the optimization problem flying in the $D$-dimensional search space, modifying (iteration by iteration) its position according to its own best position in history and that of its neighbors. Each particle $i \quad(i=1,2, \ldots, N s)$ has three attributes: its current position $x_{i, k}$, its personal best position achieved so far $x_{i, k}^{\text {pbest }}$, and its current velocity $v_{i, k}$. Note that each one of these attributes is a $D$ dimensional parameter vector. The index $k$ denotes the iteration number of the algorithm. The initial population $(k=0)$,

$$
S=\left\{x_{1,0}, x_{2,0}, \mathrm{~K}, x_{N s, 0}\right\},
$$

is taken to be uniformly distributed in the search region using the following formula:

$$
x_{i, 0}^{j}=x_{\min }+\text { random } \cdot\left(x_{\max }-x_{\min }\right) \text {, }
$$

where, $x_{i, k}^{j}$ is the position value of the $i$-th $(i=1,2, \ldots, N s)$ particle with respect to the $j$-th $(j=1,2, \ldots, D)$ dimension. $x_{\text {min }}$ and $x_{\text {max }}$ are user defined bounds, and random is a uniform random number in $(0,1)$. Similarly, the initial velocities of the particles are generated using the formula

$$
v_{i, 0}^{j}=v_{\min }+\text { random } \cdot\left(v_{\max }-v_{\min }\right),
$$

with $v_{\min }, v_{\max }$ user-defined fixed bounds, and random a uniform random number in $(0,1)$.

At each iteration $k$, all particles in $S$ are targeted for replacement. This is achieved by performing the following steps:

\section{STEP 1:}

For each particle $i(i=1,2, \ldots, N s)$

1.1) Evaluate its objective function $f\left(x_{i, k}\right)$.

1.2) Determine its personal best position $x_{i, k}^{\text {pbest }}$ as in the following:

if $k=0$ then $x_{i, 0}^{\text {pbest }}=x_{i, 0}$

$$
\text { else if } f\left(x_{i, k}\right)<f\left(x_{i, k}^{p b e s t}\right) \text { then } x_{i, k}^{\text {pbest }}=x_{i, k}
$$

\section{STEP 2:}

Determine the global best position $x_{k}^{\text {gbest }}$ corresponding to the best objective function value among the population of the particles (i.e., the whole swarm).

\section{STEP 3:}

For each particle $i \quad(i=1,2, \ldots, N s)$ update its velocity $v_{i, k}$ as in the following:

$$
\begin{aligned}
v_{i, k}= & c_{1} \cdot r_{1} \cdot\left(x_{i, k}^{\text {pbest }}-x_{i, k}\right)+c_{2} \cdot r_{2} \cdot\left(x_{k}^{\text {gbest }}-x_{i, k}\right) \\
& +w_{k} \cdot v_{i, k-1}
\end{aligned}
$$

where, $c_{1}$ and $c_{2}$ are called cognitive and social parameters, respectively, and $r_{1}, r_{2}$ are uniform random numbers drawn in $(0,1), c_{1}$ and $c_{2}$ (also known with the term learning factors) represent the attraction that a particle has toward to its own success $\left(c_{1}\right)$, or that of its neighbors $\left(c_{2}\right)$. In other words, $c_{1}$ is a weight factor representing the attraction toward $x_{i, k}^{\text {pbest }}$, while $c_{2}$ the attraction toward $x_{k}^{\text {gbest }}$. Both of them are usually defined to be constants during the execution of the algorithm. $w_{k}$ in Eq. (6) is the inertia weight factor which gadgets the effect of the old velocity onto the new one. Generally, $w_{k}$ is updated by the linear equation:

$$
w_{k}=\Theta \times w_{k-1}
$$

where, $\Theta$ is a decrement user-defined constant factor.

\section{STEP 4:}

For each particle $i \quad(i=1,2, \ldots, N s)$ calculate its new position as in the following:

$$
x_{i, k}=x_{i, k-1}+v_{i, k}, \quad \text { for } k>0
$$

\section{STEP 5:}

Repeat steps (1)-(4) until $k$ exceeds a maximum (userdefined) number of iterations.

\section{The proposed PSO algorithm for the CDD SMETSP}

\subsection{Solution space and encoding mechanisms}

The core idea behind the developed PSO algorithm is to search for solutions that are $\mathrm{V}$-shaped. This is accomplished by the following encoding scheme that designates each job either being early or tardy: assuming an $n$-job SMETSP a candidate solution $x_{i, k}$ $(i=1,2, \ldots, N s)$ denoting the position of the $i$-th particle in 
the $k$-th iteration, is a vector containing $n$ floating-point numbers taken from values within the range $[0,1]$. Each such floating-point number is associated to a specific job $1,2, \ldots, n$ with that order. A value less than or equal to 0.5 in the vector indicates that the corresponding job is early otherwise the job is tardy. So, the jobs are distinguished into two sets namely, $S_{E}$ and $S_{T}$ containing the early and the tardy jobs respectively. Following the $\mathrm{V}$-shaped property, the jobs in $S_{E}$ are moved in the start of the schedule and sequenced in 'I-shaped' format. Late jobs are moved in the end of the schedule and sequenced in '/-shaped' format. Let sump the total processing time of the early jobs in $S_{E}$, then an optimal solution to SMETSP can fall in one of the following three cases $^{18}$ :

(A) The first job in $S_{E}$ starts at time zero and the last job in $S_{E}$ finished exactly on due date $d$.

(B) The first job in $S_{E}$ starts at time zero and the last job in $S_{E}$ is completed prior to $d$. Further, a straddling job exists, i.e., a job starting executed before $d$ and ending after $d$.

(C) The first job in $S_{E}$ does not start at time zero (i.e., it is delayed) and the last job in $S_{E}$ is finished exactly on the due date $d$.

Case-(A) occurs when sump $=d$; case-(B) occurs when sump $>d$, while case-(C) occurs when sump $<d$. Therefore, according to the proposed scheme, for every candidate vector of the entire population, the sets $S_{E}$ and $S_{T}$ are firstly created. Second, the processing time of the jobs in $S_{E}$ are summed up into sump until the value of this variable surpassed $d$ or no other jobs are contained in $S_{E}$. Third, the starting time of the first job in $S_{E}$ is defined. That is, when $\operatorname{sump} \geq d$ (cases (A) and (B)), the first job starts at time zero, otherwise (case (C)), the first job is delayed starting at time $d$-sump. Fourth, jobs in $S_{E}$ are ordered based on 'I-shaped' property, while jobs in $S_{T}$ are ordered based on '/-shaped' property. This encoding mechanism is given below in algorithmic form. $\Psi=\left(\psi_{1, \ldots}, \psi_{n}\right)$ is an individual vector solution, while the notation $\{i\}$ denotes the $i$-th job $(i=1, \ldots, n)$.

Procedure Encoding_mechanism $(\Psi)$

begin

Step1: Build sets $S_{E}$ and $S_{T}$

$S_{E}=S_{T}=\varnothing ;$ sump $=0 ;$ caseB $=$ false ;

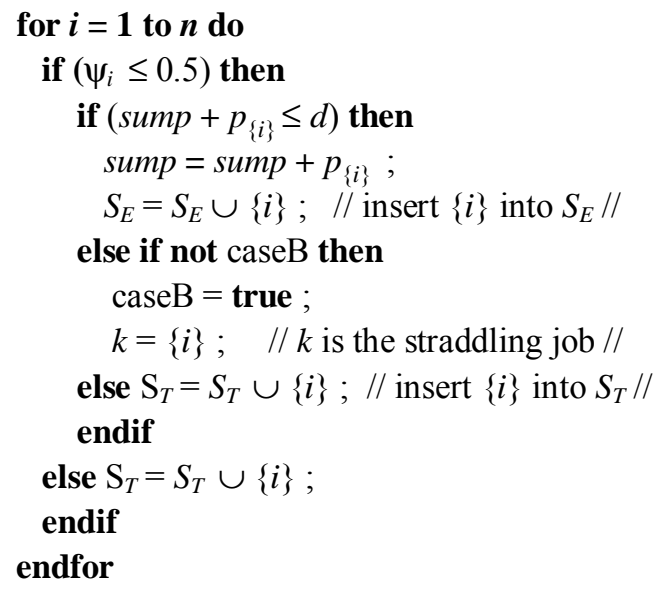

\section{Step2: Build the final schedule}

if caseB then // case-(B) //

Sort the jobs in $S_{E}$ according to 'I-shaped' format.

Then, put at the tail of $S_{E}$ the straddling job $k$. Sort the jobs in $S_{T}$ according to '/-shaped' format. First job in $S_{E}$ starts at time Tstart $=0$.

else // case-(A), or -(C) //

Sort the jobs in $S_{E}$ and $S_{T}$ according to 'V-shaped' property. First job in $S_{E}$ starts at time

Tstart $=d-$ sump

endif

$g=S_{E}+S_{T} / /$ final schedule //

Return ( $g$, Tstart)

end

Let us discuss how this mechanism works through a simple example for the 8-job SMETSP given in Table 1. The demand is to finish the jobs on a common due date $d=55$. The summation of the tasks' processing times is equal to $\sum p_{\mathrm{i}}=91 \quad(i=1, \ldots, 8)$. Suppose the following floating-point vector is given, generated at some point in time by PSO algorithm

$\Psi=(0.33,0.76,0.10,0.40,0.05,0.20,0.11,0.86)$

Table 1: Jobs' characteristics for an 8-jobs SMETSP.

\begin{tabular}{ccccccccc}
\hline & $\mathrm{j} 1$ & $\mathrm{j} 2$ & $\mathrm{j} 3$ & $\mathrm{j} 4$ & $\mathrm{j} 5$ & $\mathrm{j} 6$ & $\mathrm{j} 7$ & $\mathrm{j} 8$ \\
\hline $\mathrm{p}_{\mathrm{i}}$ & 20 & 6 & 13 & 13 & 12 & 12 & 12 & 3 \\
$\alpha_{\mathrm{i}}$ & 4 & 1 & 5 & 2 & 7 & 9 & 5 & 6 \\
$\beta_{\mathrm{i}}$ & 5 & 15 & 13 & 13 & 6 & 6 & 15 & 1 \\
$\mathrm{p}_{\mathrm{i}} / \alpha_{\mathrm{i}}$ & 5 & 6 & 2.6 & 6.5 & 1.7 & 1.3 & 2.4 & 0.5 \\
$\mathrm{p}_{\mathrm{i}} / \beta_{\mathrm{i}}$ & 4 & 0.4 & 1 & 1 & 2 & 2 & 0.8 & 3 \\
\hline
\end{tabular}

Since the $2^{\text {nd }}$ and $8^{\text {th }}$ components of $\Psi$ have values greater than 0.5 , then jobs 2 and 8 are put in the tardy 
set $S_{T}$. All the remaining jobs are candidate members of the early set $S_{E}$. However, a job $\{i\}$ is placed in $S_{E}$ only when the following three conditions are satisfied: (1) $\psi_{i} \leq 0.5$, (2) sump $+p_{1} \leq d$, and (3) $\{i\}$ is not a straddling job. Otherwise, $\{i\}$ is placed in $S_{T}$.

Hence, after applying step 1 of the above encoding mechanism, the two sets are formed as $S_{E}=\{1,3,4\}$, and $S_{T}=\{2,6,7,8\}$; while job $\{5\}$ becomes a straddling job. Therefore, the solution represented by $\Psi$ falls into case-(B), meaning that the jobs in $S_{E}$ and $S_{T}$ must be sequenced according to 'I-shaped' and '/-shaped' order, respectively. While the straddling job must be put between the tail of $S_{E}$ and the head of $S_{T}$. Using the values given in Table 1, the final V-shaped solution corresponding to $\Psi$ is displayed in Figure 2 .

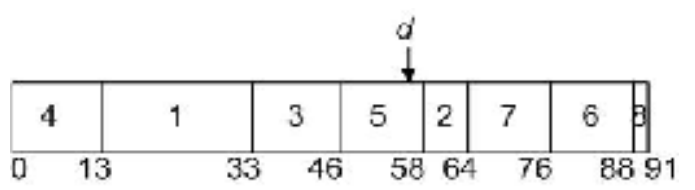

Fig. 2: The schedule for the 8 -job problem of Table 1 corresponding to the particle

\subsection{PSO implementation for the SMETSP}

Except from the above encoding mechanism which is necessary for mapping a particle's position to an actual schedule solution, in order to apply PSO to SMETSP a way must be found for using Eqs (6) and (8). To that purpose, a technique similar to that previously used by Allahverdi and Al-Anzi ${ }^{43}$ for the solution of a two-stage assembly-scheduling problem was adopted. Let us see how this technique works through a simple example:

Suppose that, for a 5-job SMETSP at some point in time during the $k$-th iteration, a specific particle say $x_{\varphi, k}(\varphi \in[1, N s])$ in the swarm has the following characteristics: $\quad$ Present $=(3,4,1,2,5)$, Pbest $=$ $(1,4,5,2,3)$, and Gbest $=(2,4,5,1,3)$. Present denotes the SMETSP solution represented by $x_{\varphi, k}$. Pbest denotes the schedule solution corresponding to $x_{\phi, k}^{\text {pbest }}$ and Gbest the schedule solution corresponding to $x_{k}^{\text {gbest }}$.

Let, $D_{l}$ be the fraction of jobs that are different between Present and Pbest; and $D_{2}$ the fraction of jobs that are different between Present and Gbest. In other words, $D_{1}$ and $D_{2}$ represent the differences between the jobs' sequences Present and Pbest, and Present and Gbest, respectively. Therefore, for the above example, since Present differs from Pbest in three locations (actually in the $1^{\text {st }}, 3^{\text {rd }}$, and $5^{\text {th }}$ locations) then $D_{l}=3 / 5=$ 0.6. Similarly, Present differs from Gbest in four (out the five in total) locations meaning that $D_{2}=4 / 5=0.8$. Eqs. (6) and (8) can now be written as in the following ${ }^{43}$ :

Advance Present towards Pbest with a velocity,

$$
V_{l}=c_{l} \cdot r_{l} \cdot D_{l}
$$

Advance Present towards Gbest with a velocity,

$$
V_{2}=c_{2} \cdot r_{2} \cdot D_{2}
$$

$r_{l}, \quad r_{2}$ are uniform random numbers drawn in $(0,1)$. Advancing Present towards Pbest at a speed equal to $V_{l}$ is implemented using the procedure:

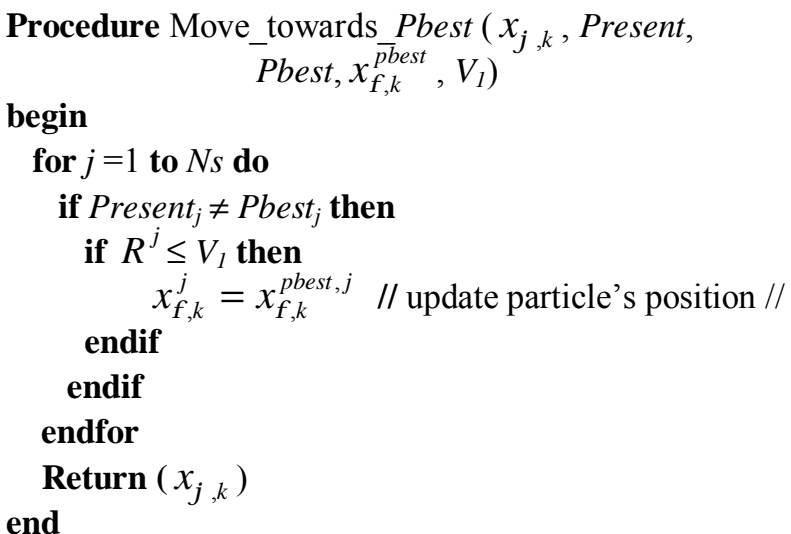

Where, Present $_{j}$ denotes the $j$-th job in Present sequence, and Pbest ${ }_{j}$ the $j$-th job in Pbest sequence. $R^{j} \in(0,1)$, is drawn randomly for each $j$. Similarly, advancing Present towards Gbest at a speed equal to $V_{2}$ is implemented using the procedure:

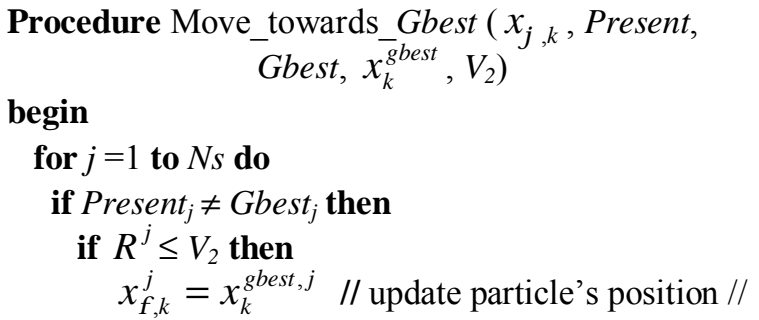




\author{
endif \\ endif \\ endfor \\ Return $\left(x_{\varphi, k}\right)$ \\ end
}

Therefore, if the job lying at position $j(j=1, \ldots, n)$ of Present is different from the associated job in Pbest (Gbest), then make the content of the $j$-th component of $x_{\varphi, k}$ same to that of the $j$-th component of $x_{k}^{\text {pbest }}\left(x_{k}^{\text {gbest }}\right)$ with probability $V_{I}\left(V_{2}\right)$. Note that, $V_{I}$ and $V_{2}$ are limited to take values in the range $(0,1)$. Possible negative values are set to 0.01 , and values greater than 1 are set to 0.95 . The complete version of the proposed PSO algorithm is given below:

\section{Algorithm PSO for the SMETSP}

Input: The number of the jobs to be scheduled $(n)$. The common due date $(d)$. Three quantities $\left(p_{j}, \alpha_{j}, \beta_{j}\right) \forall$ job $j(j=1, \ldots, n)$ corresponding to the job's processing time, and job's earliness and tardiness penalties, respectively.

Output: The 'best' V-shaped Schedule (VS*).

\section{Begin}

\section{Step 1: Initialize control parameters}

1.1) Set the size Ns of the swarm;

1.2) Set values for the weight factors $c_{l}$ and $c_{2}$;

1.3) Initialize iteration counter $k=0$, and set the maximum number of iterations $k_{M A X}$;

\section{Step 2: Swarm Initialization}

2.1) Set $x_{\text {min }}=0, x_{\text {max }}=1, v_{\text {min }}=0, v_{\text {max }}=1$;

2.2) Build initial swarm $S$ using Eq. (3).

2.3) For each particle $i(i=1,2, \ldots, N s)$ in $S$, create its initial velocity $v_{i, k}$ using Eq. (4).

\section{Step 3: Swarm Evaluation}

for $i=1$ to $N s$ do

3.1) Build schedule $v \mathrm{~s}^{x_{i, k}}$ corresponding to $x_{i, k}$

3.2) Set $v \mathrm{~s}^{x_{i, k}}=$ Encoding_mechanism $\left(x_{i, k}\right)$;

3.3) Compute the cost of the generated schedule, $\operatorname{COST}\left(v \mathrm{~s}^{x_{i, k}}\right)$ using Eq. (1);

3.4) Determine the personal best position and its associated V-shaped schedule as in the following:

$$
\begin{aligned}
& \text { if }(k=0) \text { then } \\
& \quad x_{i, k}^{\text {pbest }}=x_{i, k} ; v s_{i, k}^{\text {pbest }}=v \mathrm{~s}^{x_{i, k}} ; \\
& \text { elseif } \operatorname{COST}\left(v \mathrm{~s}^{x_{i, k}}\right)<\operatorname{COST}\left(v s_{i, k}^{\text {pbest }}\right) \text { then } \\
& \quad x_{i, k}^{\text {pbest }}=x_{i, k} ; v s_{i, k}^{\text {phest }}=v \mathrm{~s}^{x_{i, k}} ;
\end{aligned}
$$

$$
\begin{gathered}
\text { endif } \\
\text { endfor }
\end{gathered}
$$

Step 4: Population Statistics:

4.1) Determine the global best V-shaped schedule of the swarm $\left(v s_{k}^{\text {gbest }}\right)$; save the associated global best position into $x_{k}^{\text {gbest }}$;

4.2) Set Gbest $=v s_{k}^{\text {gbest }}$;

4.3) Keep track for the best-so-far V-shaped solution $v \mathrm{~s}^{*}$ :

$$
\begin{aligned}
& \text { if } k=0 \text { then } v \mathrm{~S}^{*}=v s_{k}^{\text {gbest }} \\
& \text { else if } \operatorname{COST}\left(v s_{k}^{g \text { best }}\right)<\operatorname{COST}\left(v \mathrm{~S}^{*}\right) \text { then } \\
& \quad v \mathrm{~S}^{*}=v \mathrm{~s}_{k}^{g \text { best }} \\
& \text { endif }
\end{aligned}
$$

\section{Step 5: Update velocity and position}

for each particle $i(i=1,2, \ldots, N s)$ in $S \mathbf{d o}$

5.1) Set Present $=v \mathrm{~s}^{x_{i, k}}$, Pbest $=v s_{i, k}^{\text {pbest }}$;

5.2) Determine $D_{1}$ and $D_{2}$ using the method described in sub-section 4.2;

5.3) Compute $V_{l}$ using Eq.(9), repair $V_{l}$ if needed;

5.4) Compute $V_{2}$ using Eq.(10), repair $V_{2}$ if needed;

5.5) Advance Present towards Pbest at a speed $V_{I}$ using: Move_towards_Pbest $\left(x_{i, k}\right.$, Present,

$$
\text { Pbest, } x_{i, k}^{\text {pbest }}, \bar{V}_{l} \text { ); }
$$

5.6) Advance Present towards Gbest at a speed $V_{2}$ using:Move_towards_Gbest $\left(x_{i, k}\right.$, Present,

$$
\text { Gbest, } \left.x_{k}^{\text {gbest }}, V_{2}\right) \text {; }
$$

\section{endfor}

\section{Step 6: Stopping criterion}

6.1) Advance iteration counter: $k=k+1$;

$6.2)$ if $k \leq k_{M A X}$ then go to Step 3 .

6.3) Return $\left(v s^{*}\right)$;

End;

\section{Experimental analysis and discussion}

The proposed PSO algorithm was implemented in Pascal and run on a Pentium $4(1.2 \mathrm{GHz})$ PC. The algorithm was tested over a set of public benchmarks problems, recently proposed by Biskup and Feldmann ${ }^{25}$. These benchmarks include test instances ranging from small size with 10 jobs to large size instances with 1000 jobs. Specifically, there are seven categories of problems with 10, 20, 50, 100, 200, 500, and 1000 number of jobs with each category containing ten instances to be tested. The value of a restrictive 
factor $h=0.2,0.4,0.6,0.8$ classifies the problems as less or more restricted against a common due date $d$ using the relation:

$$
d=\left\lfloor h \sum_{j=1}^{n} p_{j}\right\rfloor
$$

With $\lfloor y\rfloor$, denoting the biggest integer smaller than, or equal to $y$. That is, for each problem, the common due date $d$ is estimated by multiplying the summation of the processing times of all the $n$ jobs with the restrictive factor $h$. The lower the value of $h$ the more restrictive is $d$ (the higher the expected percentage of the late jobs). Note that $h$ must be within $(0,1)$ recognizing that, for $h=1$ the problem becomes unrestrictive, while for $h=0$ the problem becomes trivial. It is worth pointing out that optimal solutions for the examined benchmarks only exist for the 10 jobs test instances and have been achieved using an integer programming formulation with LINDO software ${ }^{25}$.

As described in section 3 the performance of the proposed PSO algorithm is depended on the values of three parameters: the swarm size $(N s)$, the cognitive parameter $\left(c_{1}\right)$, and the social parameter $\left(c_{2}\right)$. In order to determine the correct settings for these parameters, the following control schemes were studied:

(a) Different swarm sizes were examined in the range, $N s \in\{\max (10,5+[\sqrt{n}]), n, 2 n, 3 n\}$; with $n$ being the number of the jobs to be scheduled. The function $\max (\mathrm{x}, \mathrm{y})$ returns the maximum between $x$ and $y$. Note that, formula $\max (10$, $5+[\sqrt{n}]$ guarantees that $N s$ will be never less than 10 jobs (cases of test problems with $n=10$ and $n=20$ jobs). Using swarms with less than 10 particles was found in preliminary experiments to reduce drastically the performance of the developed algorithm resulting to solutions with poor quality.

(b) To be consistent with the literature ${ }^{46,47}$ the two learning factors $c_{1}$ and $c_{2}$ were both set to a fixed and equal value within the range $\{0.5,1.0,2.0\}$. Furthermore, since some recent works (e.g., see Refs. 47 and 48) report that it might be even better to choose a larger cognitive parameter, $c_{1}$, than a social parameter, $c_{2}$, but with $c_{1}+c_{2} \leq 4$, experiments have been also conducted using the combination $c_{1}=2$ and $c_{2}=1.5$.
In all the simulations, the PSO algorithm was left running for a maximum number of 500 n evaluations (where an evaluation corresponds to the estimation of the objective (cost) function of an individual solution) and the best results obtained after this time duration were reported. The particular maximum running duration was found in preliminary experiments to be a quite good choice for terminating the running of the developed algorithm. It was the main result of an empirical investigation performed with the following 5 termination conditions alternatives: stop after running for a maximum of, (a) $500 n$ evaluations, (b) $1000 n$ evaluations, (c) 1000 iterations; and (d) stop the algorithm if after a number of 50 iterations no improvement to the best so far solution is encountered.

Moreover, in order to be fair with the stochastic behavior of the algorithm, multiple runs over each test instance were performed and the average results were reported. More specifically, to get the average performance of the PSO algorithm, 15 runs (starting each time from a different random number seed) on each problem instance were performed and the solution quality was averaged. Two performance measures were used to quantify the performance of the algorithm:

(i). The average percentage deviation (dev\%) from the existing optimum solution defined by the equation:

$$
\operatorname{dev} \%=100 \times\left(\operatorname{Cost}_{P S O}-U B\right) / U B
$$

Where, Cost $_{P S O}$ is the cost (Eq.(1)) of the best schedule achieved by the PSO algorithm for a specific benchmark instance; and $U B$ (=upper bound) the corresponding cost of the existing best known solution for this benchmark instance as reported in Ref. 25 .

(ii). The average CPU time (measured in seconds) consumed over each test instance until the convergence of the algorithm.

Table 2 displays the influence of the various combinations of settings of the control parameters ( Ns, $\left.c_{1}, c_{2}\right)$ on the performance of the PSO algorithm in regard to $d e v \%$. The results displayed concern the application of the PSO algorithm over the 10 instances included in the 50-job SMETSP $(h=0.2)$ benchmarks. It is underlined that a similar influence of these 
parameters on the performance of the algorithm was also investigated over the other benchmarks categories. As one can see from Table 2, best results were encountered using the combination, $N s=\max (10$, $5+[\sqrt{n}]), c_{1}=2.0$, and $c_{2}=1.5$. The minus sign in the numerical results of this table denotes that PSO attained to generate schedules of lower costs than the costs of the existing best schedules for the specific benchmarks. Particularly, PSO improved the existing best objective function values for these benchmarks by approx. 5.5\%.

Table 2: Choosing the correct control settings for the PSO algorithm. Average dev $\%$ of the best solutions obtained after 15 runs over the instances of the 50 -jobs $(h=0.2)$ benchmarks.

\begin{tabular}{|c|c|c|c|c|}
\hline$N s$ & $\begin{array}{l}c_{1}=c_{2} \\
=0.5\end{array}$ & $\begin{aligned} c_{1} & =c_{2} \\
& =1\end{aligned}$ & $\begin{aligned} c_{1} & =c_{2} \\
& =2\end{aligned}$ & $\begin{array}{l}c_{1}=2.0 \\
c_{2}=1.5\end{array}$ \\
\hline $\max (10,5+\lfloor\sqrt{n}\rfloor)$ & -5.21 & -5.42 & -5.48 & -5.55 \\
\hline$n$ & -5.29 & -5.26 & -5.26 & -5.34 \\
\hline $2 n$ & -4.98 & -5.18 & -5.18 & -5.21 \\
\hline $3 n$ & -5.02 & -5.14 & -5.20 & -5.20 \\
\hline
\end{tabular}

In regard to the results of Table 2, one more comment about the selection of the appropriate population (swarm) size must be given for the interested reader. The empirical knowledge generally dictates that a larger population will work more slowly but will eventually achieve better solutions than a smaller population. However, this is not always true and the correct population size depends on the problem being solved, the run duration of the algorithm, the representation used and the operators manipulating this representation ${ }^{49}$. The experimental results presented throughout this paper are performed with the PSO algorithm running for a maximum number of $500 n$ evaluations. Where, an evaluation corresponds to the estimation of the objective function of an individual solution. That is, given that the PSO algorithm is going to perform $500 n=500 \times 50=25,000$ evaluations for the 50-job SMETSP, 50,000 evaluations for the 100-job SMETSP, and so on, the above preliminary tests indicated that a population of $\max (10,5+\lfloor\sqrt{n}\rfloor)$ individuals is an effective choice to deal with the specific implementation of the PSO algorithm.
The following discussion concerns the application of the PSO algorithm using the above 'best' combination of settings. The experiments have been carried out over the most difficult test instances of the Biskup and Feldmann (2001)'s benchmarks ${ }^{25}$; i.e., those instances that are more restricted against common due dates (i.e., with $h=0.2$ and 0.4 ). The full computational results obtained by the proposed PSO algorithm are summarized in Tables 3 and 4. In particular, Table 3 contains the results concerning the small size benchmark problems with up to 50 jobs; while Table 4 contains the results concerning the large size benchmarks problems with jobs ranging from 100 to 1000. For each test instance the two tables include the existing upper bound $(U B)$ generated by Biskup and Feldmann ${ }^{25}$, the near-optimum solution achieved by the PSO algorithm (Cost $t_{P S O}$ ), and the percentage offset $(d e v \%)$ of the generated solution from the existing upper bound. It is worth pointing out that optimal solutions for these benchmarks only exist for the 10 jobs test instances and have been achieved using an integer programming formulation with LINDO software ${ }^{25}$.

Table 3: Results over the small size benchmarks.

\begin{tabular}{|c|c|c|c|c|c|c|c|}
\hline & & $h=$ & 0.2 & & & 0.4 & \\
\hline & & $\mathrm{UB} /$ & & & $\mathrm{UB} /$ & & \\
\hline$n$ & & OPTIMUM & $\operatorname{Cost}_{p s i}$ & dev\% & OPTIMUM & $\operatorname{Cost}_{p s}$ & $\operatorname{dev} \%$ \\
\hline 10 & 1 & 1936 & 1936 & 0.00 & 1025 & 1025 & 0.00 \\
\hline & 2 & 1042 & 1042 & 0.00 & 615 & 615 & 0.00 \\
\hline & 3 & 1586 & 1602 & 1.01 & 917 & 931 & 1.53 \\
\hline & 4 & 2139 & 2169 & 1.40 & 1230 & 1230 & 0.00 \\
\hline & 5 & 1187 & 1187 & 0.00 & 630 & 630 & 0.00 \\
\hline & 6 & 1521 & 1521 & 0.00 & 908 & 908 & 0.00 \\
\hline & 7 & 2170 & 2170 & 0.00 & 1374 & 1374 & 0.00 \\
\hline & 8 & 1720 & 1720 & 0.00 & 1020 & 1020 & 0.00 \\
\hline & 9 & 1574 & 1574 & 0.00 & 876 & 876 & 0.00 \\
\hline & 0 & 1869 & 1869 & 0.00 & 1136 & 1140 & 0.35 \\
\hline 20 & 1 & 4431 & 4394 & -0.84 & 3066 & 3066 & 0.00 \\
\hline & 2 & 8567 & 8460 & -1.25 & 4897 & 4897 & 0.00 \\
\hline & 3 & 6331 & 6221 & -1.74 & 3883 & 3845 & -0.98 \\
\hline & 4 & 9478 & 9192 & -3.02 & 5122 & 5122 & 0.00 \\
\hline & 5 & 4340 & 4215 & -2.88 & 2571 & 2495 & -2.96 \\
\hline
\end{tabular}




\begin{tabular}{|c|c|c|c|c|c|c|}
\hline 6 & 6766 & 6552 & -3.16 & 3601 & 3584 & -0.47 \\
\hline 7 & 11101 & 10459 & -5.78 & 6357 & 6250 & -1.68 \\
\hline 8 & 4203 & 3994 & -4.97 & 2151 & 2201 & 2.32 \\
\hline 9 & 3530 & 3465 & -1.84 & 2097 & 2096 & -0.05 \\
\hline 10 & 5545 & 4987 & 10.06 & 3192 & 2925 & -8.36 \\
\hline 501 & 42363 & 40734 & -3.85 & 24868 & 23792 & -4.33 \\
\hline 2 & 33637 & 30739 & -8.62 & 19279 & 18042 & -6.42 \\
\hline 3 & 37641 & 34505 & -8.33 & 21353 & 20700 & -3.06 \\
\hline 4 & 30166 & 27803 & -7.83 & 17495 & 16693 & -4.58 \\
\hline 5 & 32604 & 32332 & -0.83 & 18441 & 18167 & -1.49 \\
\hline 6 & 36920 & 35102 & -4.92 & 21497 & 20402 & -5.09 \\
\hline 7 & 44277 & 43229 & -2.37 & 23883 & 23228 & -2.74 \\
\hline 8 & 46065 & 43969 & -4.55 & 25402 & 24947 & -1.79 \\
\hline 9 & 36397 & 34326 & -5.69 & 21929 & 20008 & -8.76 \\
\hline 10 & 35797 & 32999 & -7.82 & 20048 & 19238 & -4.04 \\
\hline
\end{tabular}

Table 4: Results over the large size benchmarks.

\begin{tabular}{|c|c|c|c|c|c|c|c|}
\hline & & \multicolumn{3}{|c|}{$h=0.2$} & \multicolumn{3}{|c|}{$h=0.4$} \\
\hline$\underline{n}$ & \multicolumn{2}{|r|}{$\begin{array}{c}\text { UB/ } \\
\text { OPTIMUM }\end{array}$} & $\operatorname{Cost}_{p s o}$ & $\operatorname{dev} \%$ & $\begin{array}{c}\text { UB/ } \\
\text { DPTIMUN }\end{array}$ & $\operatorname{Cost}_{p s c}$ & ${ }_{2} \operatorname{dev} \%$ \\
\hline \multirow[t]{10}{*}{100} & 1 & 156103 & 146132 & -6.39 & 89588 & 86280 & -3.69 \\
\hline & 2 & 132605 & 125331 & -5.49 & 74854 & 73459 & -1.86 \\
\hline & 3 & 137463 & 130414 & -5.13 & 85363 & 80207 & -6.04 \\
\hline & 4 & 137265 & 131132 & -4.47 & 87730 & 79947 & -8.87 \\
\hline & 5 & 136761 & 124882 & -8.69 & 76424 & 71609 & -6.30 \\
\hline & 6 & 151938 & 139961 & -7.88 & 86724 & 77987 & -10.07 \\
\hline & 7 & 1416 & 1374 & -2 & 798 & 78410 & -1.81 \\
\hline & 8 & 168086 & 161 & -3.96 & 95361 & 95612 & 0.26 \\
\hline & 9 & 125153 & 118859 & -5.03 & 73605 & 69812 & -5.15 \\
\hline & 10 & 124446 & $119^{\circ}$ & -3.74 & 99 & 72389 & -0.01 \\
\hline \multirow[t]{8}{*}{200} & 1 & 526666 & 502920 & -4.01 & 301449 & 298080 & -1.12 \\
\hline & 2 & 566643 & 556476 & -1.79 & 335714 & 322705 & -3.88 \\
\hline & 3 & 529919 & 497396 & -6.14 & 308278 & 296023 & -3.98 \\
\hline & 4 & 603709 & 599074 & -0.77 & 360852 & $3561 \mathrm{C}$ & -1.29 \\
\hline & 5 & 547953 & 524601 & -4.26 & 322268 & 306819 & -4.79 \\
\hline & 6 & 502276 & 490165 & -2.41 & 292453 & 287009 & -1.86 \\
\hline & 1 & 479651 & 470485 & -1.91 & 279576 & 275805 & -1.35 \\
\hline & 8 & 530896 & 505432 & -4.80 & 288746 & 280383 & -2.90 \\
\hline
\end{tabular}

\begin{tabular}{rrrrr|rrr}
9 & 575353 & 545248 & -5.23 & 331107 & 315441 & -4.73 \\
10 & 572866 & 544871 & -4.89 & 332808 & 327299 & -1.66 \\
500 & 1 & 3113088 & 2995837 & -3.77 & 1839902 & 1809150 & -1.67 \\
2 & 3569058 & 3396297 & -4.84 & 2064998 & 2019880 & -2.18 \\
3 & 3300744 & 3114316 & -5.65 & 1909304 & 1877994 & -1.64 \\
4 & 3408867 & 3243457 & -4.85 & 1930829 & 1923249 & -0.39 \\
5 & 3377547 & 3127020 & -7.42 & 1881221 & 1824148 & -3.03 \\
6 & 3024082 & 2807257 & -7.17 & 1658411 & 1637076 & -1.29 \\
7 & 3381166 & 3193413 & -5.55 & 1971176 & 1931460 & -2.01 \\
8 & 3376678 & 3127525 & -7.38 & 1924191 & 1829059 & -4.94 \\
9 & 3617807 & 3376250 & -6.68 & 2065647 & 1995866 & -3.38 \\
10 & 3315019 & 3137808 & -5.35 & 1928579 & 1867701 & -3.16 \\
1000 & 1 & 15190371 & 14496459 & -4.57 & 8570154 & 8165112 & -4.73 \\
2 & 13356727 & 12637411 & -5.39 & 7592040 & 7324309 & -3.53 \\
3 & 12919259 & 12424534 & -3.83 & 7313736 & 7057780 & -3.50 \\
4 & 12705290 & 12332609 & -2.93 & 7300217 & 7063524 & -3.24 \\
5 & 13276868 & 13013517 & -1.98 & 7738367 & 7401864 & -4.35 \\
6 & 12236080 & 11683666 & -4.51 & 7144491 & 7012026 & -1.85 \\
7 & 14160773 & 13299602 & -6.08 & 8426024 & 7898469 & -6.26 \\
8 & 13314723 & 12321242 & -7.46 & 7508507 & 7264332 & -3.25 \\
9 & 12433821 & 11789909 & -5.18 & 7299271 & 7117968 & -2.48 \\
10 & 13395234 & 12471333 & -6.90 & 7617658 & 7330962 & -3.76 \\
\hline
\end{tabular}

As one can see from Tables 3 and 4 the performance of the PSO algorithm is of high quality. The algorithm reached the exact optimum solution in the majority of the test instances with 10-jobs (see Table 3); particularly, in the eight out the ten in total instances. Furthermore, (except from three instances of the $h=0.4,20$-job benchmarks -see Table 3-) higher quality solutions than the existing best known solutions have been generated by PSO to all the test instances ranging from 20 to 1000 jobs. This fact is indicated by a negative percentage deviation (dev\%) in the corresponding columns of the two tables. More specifically, for the most restricted class of benchmarks $(h=0.2)$ the mean $\operatorname{dev} \%$ for the solutions obtained are $3.55 \%$ for 20 jobs problems, $-5.48 \%$ for 50 -jobs problems (Table 3), $-5.38 \%$ for 100 -jobs, $-3.67 \%$ for 200-jobs (Table 4), and so on. PSO introduced new upper bounds in all the $h=0.2$ instances with $n \geq 20$, i.e. to approx. $86 \%$ of the instances of $h=0.2$ benchmarks. While, in regard to the $h=0.4$ benchmarks, PSO attained 
to improve nearly the $75 \%$ of the available test instances. Figure 3 illustrates the average percentile improvement of the benchmarks solutions attained by the PSO algorithm.

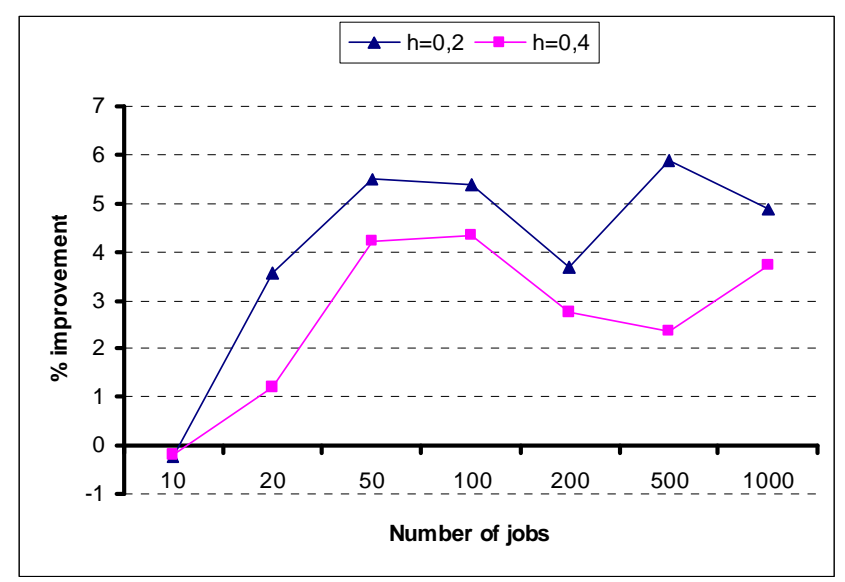

Fig. 3: Average percentile improvement of the benchmarks solutions using the PSO algorithm.

Figure 4 displays the mean actual running times for the PSO algorithm until the convergence to the nearoptimum solution. The running times (in seconds) are given in regard to the problems' degree of restriction (value of parameter $h$ ) and problems' sizes.

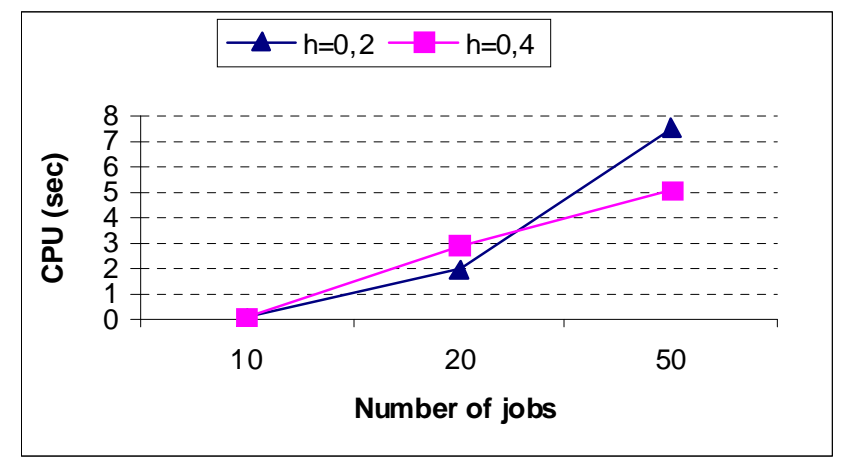

(a)

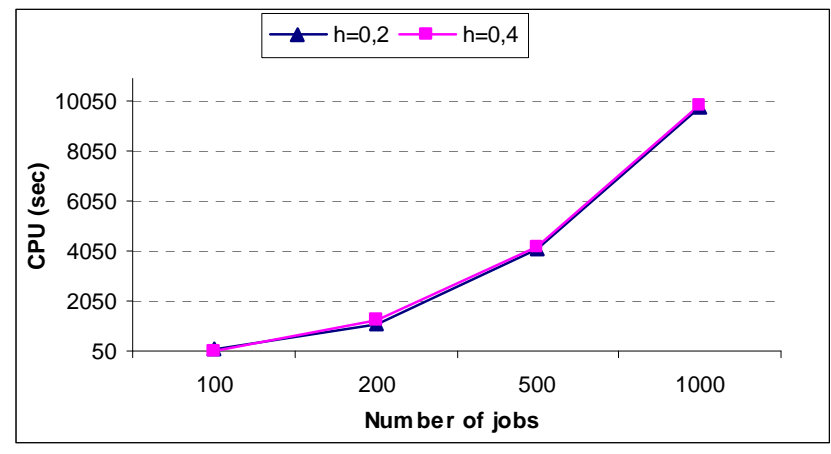

(b)

Fig. 4: Average CPU times (in seconds) until the convergence. Results on a Pentium 4 (1.2 GHz) PC. (a) case of small size problems, (b) case of large size problems.

Figure 5 displays the optimal solutions (in Gantt charts) obtained by PSO for the first two instances included in the $h=0.210$-jobs benchmarks problems. $\mathrm{f}^{*}$ denotes the cost (given by Eq. (1)) of the optimal solution. From Table 3 one can observe that PSO did not find the exact optimum solution in 4 (out the 20 in total) instances of the 10-jobs test beds. However, it is worth pointing out that, with a slight modifications of the swarm's size (particularly, with a swarm's size equal to $4 n$ which in the case of the 10-jobs problems means 40 particles in the swarm), PSO attained to determine the exact optimal solution for these 4 instances too.

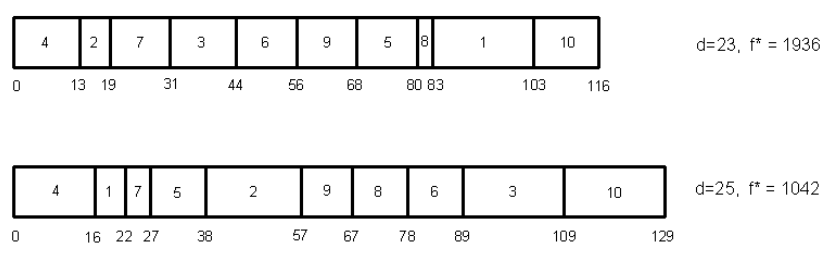

Fig. 5: The Gantt charts for the optimal solutions obtained by PSO for the first two instances included in 10 -jobs $(h=0.2)$ benchmarks problems. $\mathrm{d}$ and $\mathrm{f}^{*}$ denotes the common due-date and the cost of the optimal solution, respectively.

Some interesting comparative results between PSO and three known heuristics namely, evolution strategy (ES), stochastic hill-climber (SHC), and pure random search (pRS) technique are depicted in Fig. 6. These 
results concern the dev\% of the solutions obtained by the four methods (averaged after 15 runs) on the test instances included in the 50-jobs data sets. As one can observe from this figure, PSO (lower curve) outperforms all the other heuristics generating solutions of much higher quality (schedules of lower costs). As it was expected, the next best performance was due to ES, while the worst performance was due to $\mathrm{pRS}$ technique. The basic characteristics of the three implemented heuristics are briefly described below.

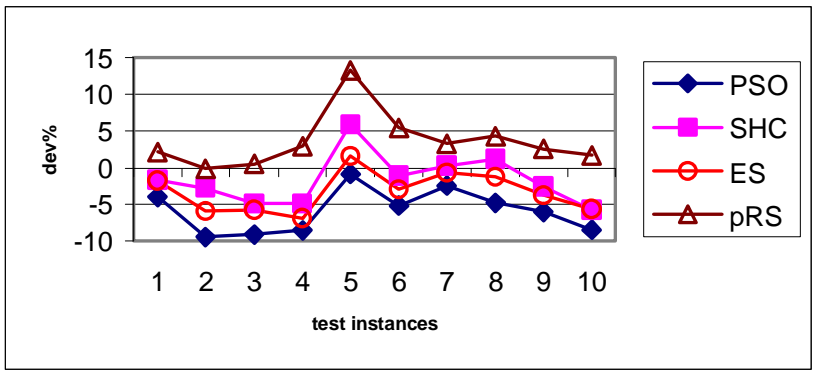

(a)

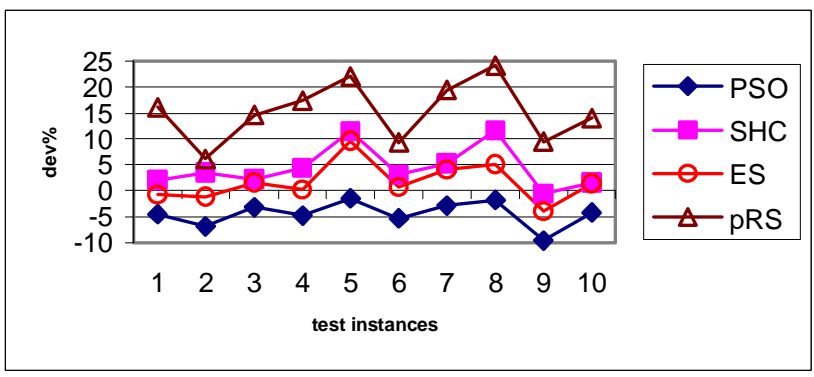

(b)

Fig. 6: Comparative results between PSO, ES, SHC and pRS over the 10 test instances included in the 50-jobs benchmarks data set. The $\%$ deviation of the solutions obtained by the four heuristics from the existing near-optimal benchmark solutions. Case of, (a) h=0.2 and (b) $h=0.4$ tests instances.

To make the three heuristics comparable to PSO we used the same population size for all, and limit the running process for the same maximum number of iterations. Moreover, all the heuristics were implemented using the (same with PSO) encoding, and evaluation mechanisms described in sub-section 4.1. Particularly, each individual solution in ES, SHC and pRS is a vector containing $n$ floating-point numbers within the range $[0,1]$. Each such floating-point vector is mapped to an actual SMETSP solution using procedure Encoding_mechanism also described in subsection 4.1. ES was implemented by following the recommendations of the literature ${ }^{50}$. After experimentation for choosing the correct survivor selection scheme, we found that using $(\mu, \lambda)$-scheme within ES results in a superior optimizer to that of using $(\mu+\lambda)$-scheme. For this reason $(\mu, \lambda)$-scheme was adopted. $\mu$ was estimated using the heuristic rule $\lambda / \mu=7$. Mutation was performed through Gaussian perturbation. Offspring were generated using discrete recombination. SHC was implemented as a modified version of the metropolis algorithm ${ }^{51}$ for a parallel searching of the solution space. That is, for each solution $x_{i, k} \in S$ in iteration $k$, search its neighbourhood $N\left(x_{i, k}\right)$ and choose the best solution $x_{i, k}^{\prime}$. Accept this solution (i.e., $\left.x_{i, k}=x_{i, k}^{\prime}\right)$ if $\operatorname{COST}\left(x_{i, k}^{\prime}\right)<\operatorname{COST}\left(x_{i, k}\right)$ or with a probability $\exp \left(\left(\operatorname{COST}\left(x_{i, k}\right)-\operatorname{COST}\left(x_{i, k}^{\prime}\right)\right) / \mathrm{T}\right) . \quad T>0 \quad$ (called temperature) determines the probability of accepting worse solutions. A value of $T=10$ was experimentally found to be a good choice for our problem. A new solution in $N\left(x_{i, k}\right)$ was generated by flipping randomly each time the $j^{\text {th }}(j=1, \ldots, n)$ component of $x_{i, k}$. Thus, for each solution we generate $n$ different neighbours. pRS is identical to SHC except from the point that, a new solution $x_{i, k}^{\prime}$ is accepted and replaced its ancestor $x_{i, k}$ only if it has a lower cost.

We conclude by giving a short guide to those of the researchers who are willing to apply PSO on their discrete COPs: (a) Select an appropriate representation. This representation is directly depended on the structure of the problem being solved. (b) Design a suitable encoding mechanism that maps each vector solution to an actual COP's solution. This mechanism must take into account the properties of the problem being solved. (c) If necessary, implement appropriate repairing mechanisms to guarantee the creation of legal solutions. (d) Follow the recommendation of the literature to estimate the proper settings of the control parameters (i.e., swarm size, inertia weight, learning factors). (e) Study the behavior of your PSO at different iterations so that to realize whether it converges too early. There are some important tricks in the literature to improve the rate of convergence of PSO, to control the diversity of the swarm so that to avoid local optima, etc. 


\section{Conclusion}

In today production management, scheduling against common due dates with respect to earliness and tardiness penalties is of great importance mainly due to its compliance with the principles of just-in-time 'philosophy'. This is a typical discrete combinatorial optimization problem (COP) known to be intractable, meaning that, the search for optimal schedules using traditional mathematical programming techniques is only possible for very small size instances of the problem.

This paper presented a new particle swarm optimization (PSO) heuristic to address the problem of scheduling a number of jobs on a single machine against a restricted common due date. It is worth noting that PSO-based heuristics have rarely been applied to discrete COPs. The developed PSO differs from the existing PSO approaches in two main points: first in the way the 'particles' are represented and mapped into actual scheduling solutions; and second, in the way of controlling the 'particles' velocities of the entire swarm. Extensive experiments were performed over 140 highly restricted against a common due date benchmarks problems for which the upper bounds were known. The results obtained showed a high quality performance for the proposed PSO heuristic introducing to the majority of the benchmark instances new upper bounds. In particular, PSO reached or surpassed the $95 \%$ of the existing optimal benchmark solutions introducing new upper bounds in the $82 \%$ of the benchmarks instances.

Furthermore, comparative experiments with existing heuristics (including evolution strategy, stochastic hillclimber, and random search) showed a substantially superior performance for the developed approach in terms of solution quality. The method is quite general and can be rather easily modified and applied to any other COP. The only modifications required to be done is, firstly in the encoding mechanism (that is mapping the particles to actual solutions to the considered problem), and secondly in the evaluation mechanism (i.e. the objective function which is problem depended). Moreover, due to the small number of control parameters used within the algorithm, parameters' tuning is performed easily. A limitation however of the proposed method is its convergence speed. In its current form the algorithm is rather slow when the number of the jobs to be scheduled exceeds 200 .

Future work will be focused on the development of a more robust and faster version of the PSO algorithm to address other more difficult scheduling problems that are known to be intractable, such as the job-shop scheduling problems. Hybridization of the PSO algorithm with local search techniques such as tabusearch or/and simulated annealing is a very promising area of research and may result in more powerful heuristic. On going research is focused to the design of a multi-objective PSO algorithm to address the multicriteria flow-shop scheduling problem. Three criteria are currently under investigations with the objective to be simultaneously minimized, namely, the makespan, the maximum tardiness and the total flow time of the jobs.

\section{References}

1. K. Baker and G. Scudder, Sequencing with earliness and tardiness penalties: A review, Operations Research 38 (1990) 22-36.

2. S. French, Sequencing and scheduling, an introduction to the mathematics of the job-shop (Ellis Horwood publication, 1990).

3. J.J. Kanet, Minimizing the average deviation of job completion times about a common due date, Naval Research Logistics 28 (1981) 643-651.

4. N. Hall, Single and multiple processor models for minimizing completion time variance, Naval Research Logistics Quarterly 33 (1986) 49-54.

5. U. Bagchi, R.S. Sullivan, and Y.L Chang. Minimizing mean absolute deviation of completion times about a common due-date, Naval Research Logistics Quarterly 33 (1986) 227-240.

6. U. Bagchi, R.S. Sullivan, and Y.L. Chang, Minimizing absolute and squared deviations of completion times with different earliness and tardiness penalties about a common due-date, Naval Research Logistics Quarterly 34 (1987) 739-751.

7. N. Hall and M. Posner, Earliness-tardiness scheduling problems I: weighted deviation of completion times about a common date, Operations Research 39 (1991) 836-846.

8. P. De, J.B Ghosh, C.E. Wells, Solving a generalized model for $\mathrm{CON}$ due date assignment and sequencing, Int. Journal of Production Economics 34 (1994) 179-185.

9. J.A. Hoogeveen, and S.L. van de Velde, Earlinesstardiness scheduling around almost equal due date, INFORMS Journal on Computing 9 (1997) 92-99. 
10. T. Cheng and M. Gupta, Survey of scheduling research involving due date determination decision, European Journal of Operational Research 38 (1989) 156-166.

11. V. Gordon, J.-M.Proth, and C. Chu, A survey of the state-of-the-art of common due date assignment and scheduling research, European Journal of Operational Research 139 (2002) 1-25.

12. T. Abdul-Razaq and C.N. Potts, Dynamic programming state-space relaxation for single-machine scheduling, Journal of the Operational Research Society 39 (1988) 141-152.

13. J.P. Souza and L.A. Wolsey, A time indexed formulation of non-preemptive single machine scheduling problems, Mathematical Programming 54 (1992) 353-367.

14. M.-T. Almeida and M. Centeno, A composite heuristic for the single machine early/tardy job scheduling problem, Computers and Operations Research 25 (1998) 625-635.

15. C.-Y. Lee and S.J. Kim, Parallel genetic algorithms for the earliness-tardiness job scheduling problem with general penalty weights, Computers and Industrial Engineering 28 (1995) 231-248.

16. Q. Hao, Z. Yang, D. Wang, Z. Li, Common due date determination and sequencing using tabu search, Computers and Operations Research 23 (1996) 409-417.

17. R.J.W. James, Using tabu search to solve the common due date early/tardy machine scheduling problem, Computers and Operations Research 24 (1997) 199-208.

18. M. Feldmann and D. Biskup, Single-machine scheduling for minimizing earliness and tardiness penalties by metaheuristic approaches, Computers and Industrial Engineering 44 (2003) 307-323.

19. A.C. Nearchou and S.L. Omirou, Differential evolution for sequencing and scheduling optimization, Journal of Heuristics 12 (2006) 395-411.

20. A.C. Nearchou, A differential evolution approach for the common due date early/tardy job scheduling problem, Computers \& Operations Research 35 (2008) 13291343.

21. Z.-J. Lee, C.-C. Chuang and K.-C. Ying, An intelligent algorithm for scheduling jobs on a single machine with a common due date, Lecture Notes in Computer Science (including subseries Lecture Notes in Artificial Intelligence and Lecture Notes in Bioinformatics) 4693 LNAI (PART 2) (2007) 689-695.

22. Z.-J. Lee, S.-W. Lin, K.-C. Ying, A dynamical ant colony optimization with heuristics for scheduling jobs on a single machine with a common due date, Studies in Computational Intelligence 128 (2008) 91-103.

23. R. M'Hallah and A. Alhajraf, Ant Colony Optimization for the Single Machine Total Earliness Tardiness Scheduling Problem, in New Frontiers in Applied Artificial Intelligence: Lecture Notes in Computer Science, N.T. Nguyen et al. (Eds.) 5027 (2008) 397-407.
24. M. Reisi and G. Moslehi, Minimizing the number of tardy jobs and maximum earliness in the single machine scheduling using an artificial immune system, Int Journal of Advanced Manufacturing Technology 54 (2011) 749-756

25. D. Biskup and M. Feldmann, Benchmarks for scheduling on a single machine against restrictive and unrestrictive common due dates, Computers and Operations Research 28 (2001) 787-801.

26. R.C. Eberhart and J. Kennedy, A new optimizer using particle swarm theory, in Proc. of the $6^{\text {th }}$ Int. Symposium on Micro Machine and Human Science, (1995) 39-43.

27. Anghinolfi and M. Paolucci, A new discrete particle swarm optimization approach for the single-machine total weighted tardiness scheduling problem with sequence-dependent setup times, European Journal of Operational Research 193 (2009) 73-85.

28. C. Low, C.-J. Hsu, C.-T. Su, A modified particle swarm optimization algorithm for a single-machine scheduling problem with periodic maintenance, Expert Systems with Applications 37 (2010) 6429-6434.

29. Q.-K. Pan, M.F. Tasgetiren and Y.-C. Liang, A discrete particle swarm optimization algorithm for single machine total earliness and tardiness problem with a common due date. In: Proc. of the World Congress on Evolutionary Computation (CEC'06, Vancouver, Canada 2006) 3281-3288.

30. T.C.E. Cheng and H.G. Kahlbacher, A proof for the longest/job/first policy in one/machine scheduling, Naval Research Logistics 38 (1991) 715-720.

31. T.-On Ting, M.V.C. Rao, C.K. Loo and S.S. Ngu, Solving Unit Commitment Problem Using Hybrid Particle Swarm Optimization, Journal of Heuristics 9 (2003) 507-520.

32. M. Clerc, Discrete particle swarm optimization, illustrated by the traveling salesman problem, New Optimization Techniques in Engineering, (Heidelberg, Germany, Springer, 2004) 219-239.

33. Salman, I. Ahmad, S. Al-Madani, Particle swarm optimization for task assignment problem, Microprocessors and Microsystems 26 (2003) 363-371.

34. G.C. Onwubolu and M. Clerc, Optimal operational path for automated drilling operations by a new heuristic approach using particle swarm optimization, Int. Journal of Production Research 42 (2004) 473-491.

35. S. Liu, J. Tang and J. Song, Order-planning model and algorithm for manufacturing steel sheets, Int. Journal of Production Economics 100 (2006) 30-43.

36. A.W. Mohemmed, N. C. Sahoo, T. K. Geok, Solving shortest path problem using particle swarm optimization, Applied Soft Computing 8 (2008) 1643-1653.

37. K. Rameshkumar, R.K. Suresh, and K.M. Mohanasundaram, Discrete particle swarm optimization (DPSO) algorithm for permutation flowshop scheduling to minimize makespan, in Proc. ICNC 2005, L. Wang, 
K. Chen, and Y.S. Ong (Eds.), Springer-Verlag, Berlin 2005) 572-581.

38. C.-J. Liao, C.-T. Tseng, P. Luarn, A discrete version of particle swarm optimization for flowshop scheduling problems, Computers \& Operations Research 34 (2007) 3099-3111.

39. Z. Lian, X. Gu and B. Jiao, A novel particle swarm optimization algorithm for permutation flow-shop scheduling to minimize makespan, Chaos, Solitons \& Fractals 5 (2008) 851-861.

40. C.-T. Tseng and C.-J. Liao, A discrete particle swarm optimization for lot-streaming flowshop scheduling problem, European Journal of Operational Research, 191 (2008) 360-373.

41. Zhang, J. Sun, X. Zhu and Q. Yang, An improved particle swarm optimization algorithm for flowshop scheduling problem, Information Processing Letters 108 (2008) 204-209.

42. M.F. Tasgetiren, Y.-C. Liang, M. Sevkli and G. Gencyilmaz, Particle swarm optimization and differential evolution for the single machine total weighted tardiness problem, Int. Journal of Production Research 44 (2006) 4737-4754.

43. Allahverdi and F.S. Al-Anzi, Evolutionary heuristics and an algorithm for the two-stage assembly scheduling problem to minimize makespan with setup times, Int. Journal of Production Research 44 (2006) 4713-4735.

44. Lei, A Pareto archive particle swarm optimization for multi-objective job shop scheduling, Computers \& Industrial Engineering 54 (2008) 960-971.

45. T.-L. Lin, S.-J. Horng, T.-W. Kao, Y.-H. Chen, R.-S. Run, R.-J. Chen, J.-L. Lai and I-H. Kuo, An efficient job-shop scheduling algorithm based on particle swarm optimization, Expert Systems with Applications 37 (2010) 2629-2636.

46. J. Kennedy, The behavior of particles, In: Porto V.W., Saravanan N, Waagen D. and Eiben A.E. (eds) Evolutionary Programming VII (Springer 1998) 581590.

47. K.E. Parsopoulos and M.N. Vrahatis, Recent approaches to global optimization problems through particle swarm optimization, Natural Computing 1 (2002) 235-306.

48. Carlisle and G. Dozier, An Off-The-Shelf PSO, in Proc. of the Particle Swarm Optimization Workshop 2001, pp. $1-6$

49. Z. Michalewicz and D.B Fogel, How to solve it: Modern heuristics, (Springer-Verlag, Berlin 2000).

50. A.E. Eiben and J.E. Smith, Introduction to evolutionary computing, (Springer, Berlin 2003).

51. N. Metropolis, A. Rosenbluth, M. Rosenbluth, A. Teller and E. Teller, Equation of state calculations for fast computing machines, Journal of Chemical Physics 21 (1953) 1087-1092. 